\title{
Doctors and the Net: caught-up or great escape?
}

\author{
Bernard Lim
}

'But what about a blood plug, doctor?'

I had just finished performing a lumbar puncture on a young patient and was explaining to his mother that a post-lumbar puncture headache was quite common.

'I read on the Internet that a blood plug can reduce the chances of having a headache after a lumbar puncture,' the mother offered enthusiastically.

'I do not think that it will be necessary. It ...'

'But the Internet said ...'

And so the conversation, or more appropriately, argument, went. In the end, the young patient did not have a blood plug and the mother left in a huff, leaving me to consider the real possibility that I might have to spend the next couple of days drafting my report about her complaint of negligence.

This was my rather abrupt baptism by fire into the world of the Internet. So I delved a little more into it and discovered a bewildering parallel universe populated by a kaleidoscopic array of information.

In America, medicine has kept pace with the Internet explosion in an intimate tango that has left the rest of the world gasping with envious admiration. Surgeons in Texas are performing operations via an Internet link to a remote robotic arm set up in a hospital in Thailand, bringing state of the art technical virtuosity to developing countries. This technology also allows procedures to be performed in remote areas on patients who might otherwise die in transit. Rural primary care practices in Kansas benefit from tertiary expertise, courtesy of an Internet link-up. Electrocardiographs, photo images and radiographs are beamed within seconds to specialists hundreds of miles away. The remote capabilities conferred by the Internet have enabled physicians on the ground to treat patients in aircraft with life threatening emergencies. Special clinics where patients can have consultations over the Internet have been set up in Singapore. Patients have investigations, like blood samples and radiology, carried out by external laboratories and can input these results into an Internet database. The doctor on the other end of the line has instant access to all current and previous investigations and is able to call up X-rays at the touch of a button. Prescriptions can be sent over the net. A panel of specialists in a wide variety of fields supervises the medical management, to ensure up to date and accurate treatment. In Georgia, USA, elderly patients with limited mobility receive daily checkups through a telemedicine linkup via the Internet. A doctor can 'examine' patients in their own homes through a wide range of tests such as electrocardiograms, blood pressure, auscultation and pulse oximetry using telemedical equipment. Indeed, remote technology has bestowed an omnipresence on the physician that is unprecedented.

In Britain, Internet patient help groups have carved a valuable niche in the management of disease. A patient in the diabetic clinic astounded me when I told him that due to a technical fault, I could not bring his HbAlc up on my computer. $\mathrm{He}$ brought up his own charts and tables documenting his glucose levels. There were graphical depictions illustrating dips and spikes in his glucose levels. Apparently, the template to do this had been set up on the Internet; all he had to do was type in the numbers. There is even a diabetic chat line where patients can exchange valuable health tips and veteran diabetics can offer their life-long experience in their battle with diabetes to diabetic virgins. Needless to say, I was dazzled.

The National University of Singapore has set up an impressive infrastructure of patient information networks. The NUS-NCI CANCERNET provides valuable information and support for cancer patients and performs a pivotal role as a surrogate for busy and overworked doctors who might not be able to give patients the whole picture. Also on the Internet is the Poisons Information database. The speed and accuracy of the service, combined with the ease with which vital information can be had, compares favourably with the standard practice of calling up the poisons center where the wait for drug information averages between half an hour to an hour.

The evolution of the Internet has given life to the concept of evidence-based medicine. For years, doctors have referred to tomes of literature in the library to help them with medical decisions and management. Hours of ploughing through catalogues and shelves for journals and articles that, more often than not, are not available, made this an exercise only for the most dedicated. One colleague
Bernard Lim MBchB MRCP, British Heart Foundation Clinical Research Fellow, Department of Cell \& Developmental Biology, University of Pennsylvania

This paper won the Teale Essay Prize for Trainees 2001

Clin Med JRCPL 2001; 1:505-6 
had a patient admitted to him with acute hepatitis. The patient was a young bodybuilder who had been taking a new anabolic steroid that was not even on the database of the drug information centre. My computer-savvy colleague searched the Internet and found two reports from Germany detailing the course and clinical features of the hepatitis associated with the anabolic steroid, as well as treatment. The patient recovered and the consultant was suitably impressed with the initiative taken by my computer literate colleague.

Transplanting medical records from the body of papyrus into virtual cyberspace has freed many doctors from the mundane hell of searching for lost results slips, misplaced radiographs, and fallen out pieces of medical notes, and has ensured the survival of a generation of grateful rainforest trees. Clinical data stored in this way can then be readily accessible from other hospitals without having to waste valuable time waiting for the vital information to arrive by taxi. Statistical information can be made available via a health and population database to epidemiologists and health officials allocating health resources. Doctors undertaking research will enjoy a welcome escape from the dusty dungeons of the hospital underworld that constitute the medical records department. Indeed, medical researchers will have access to patient data from a bewildering network of hospitals, resulting in research that is more applicable to a wider population group and not just to a particular geographical location, eradicating huge sources of bias in their research. Such a system is in place and working very well at the Mayo Clinic in America.

Medical research publication has naturally benefited from the Internet. Peer-reviewed research can now be published on the web in as little as two days where as currently it can be between six months to a year from submission to publication of a paper. The tremendous amount of time saved means that many a would-be writer would be spared the agony of a rival research group having similar research published first, due to unforeseen delays.

Teleconferencing via the Internet means that a doctor can present his research to clinicians in Brazil in the morning, discuss some of the results with professors in China in the afternoon, go over the implications and applications of the work with collaborators in Australia in the evening and still be back home in time for tea with his family.
Indeed the world of the Internet does look utopian, a fragile, nebulous oasis in a desert of technophobia and conformist traditionalism. Its detractors have argued about the ever present issue of confidentiality. The computer hackers who have made their presence felt have made this a real and worrying issue and the possibility of hacking is the Achilles' heel of the Internet. Unscrupulous medical insurance companies can tap into the mercenary tendencies of these hackers to weed out potential high claimers. The prospect of getting the wrong diagnosis over the Internet has prompted the ubiquitous presence of disclaimers preceding the Internet patient information network web page. Indeed the ease with which information can be downloaded onto the web means that medical cowboys and mavericks can unleash unproven and sometimes dangerous treatment onto an unsuspecting public. Viagra, the wonder drug for impotence, attracted notoriety when it was made available over the Internet, after reports of myocardial infarcation in susceptible unscreened patients were publicised. The legal ramifications of the Internet thus circle like a vulture waiting to devour the unwary doctor. The advent of the computer virus has opened up the medical community to the threat of international 'e-terrorism'. The nightmare scenario of a virus running amok in the virtual filing cabinets of the clinical patient database, chewing up precious information, deleting and mixing up files is real and would indeed be one virus for which many a doctor would be grateful for an electronic vaccine.

The Internet thus represents a portal where a doctor can escape from the routine boredom of paperwork and tedious bureaucracy. More than ever, the feeling of the doctor having deity-like status has been reinforced by the remote presence conferred by the Internet. Rapid dissemination of medical knowledge and expertise through the Internet has reduced the disparity in medical care between developed and developing countries. Against this euphoria must be tempered the security and legal issues that have struggled to keep pace with the explosive momentum of the juggernaut that is the Internet.

Address for correspondence: Dr Bernard Lim, c/o Dr John Weisel, 1042 Department of Cell and Developmental Biology, University of Pennsylvania, 421 Curie Boulevard, BRB 11/111, Philadelphia, PA 19104, USA 